Díaz, D., Velásquez Sánchez, M. I., Rincón Barreto, D. M., Blanco Belén, O. A., y Correa López, R. A. (enero-abril, 2022). Relación entre rasgos de personalidad, toma de decisiones y la permanencia académica. Revista Virtual Universidad Católica del Norte, (65), 263-283.

$$
\text { https://www.doi.org/10.35575/rvucn.n65a10 }
$$

\title{
Relación entre rasgos de personalidad, toma de decisiones y la permanencia académica
}

Relationship between personality traits, decision-making and academic permanence

\section{Daniela Díaz}

Estudiante de psicología

Facultad de Psicología y Ciencias Sociales, Universidad Católica Luis Amigó

Medellín, Colombia

daniela.diazdi@amigo.edu.co

Orcid: https://orcid.org/0000-0002-1650-6220

CvLAC:

https://scienti.minciencias.gov.co/cvlac/visualizador/generarCurriculoCv.do?cod_rh=000178701 $\underline{8}$

\section{María Isabel Velásquez Sánchez}

Estudiante de psicología

Facultad de Psicología y Ciencias Sociales, Universidad Católica Luis Amigó

Medellín, Colombia

maria.velasqueznc@amigo.edu.co

Orcid: https://orcid.org/0000-0003-1035-2491

CvLAC:

https://scienti.minciencias.gov.co/cvlac/visualizador/generarCurriculoCv.do?cod_rh=000178619 $\underline{4}$

\section{Dubis Marcela Rincón Barreto}

Doctoranda en Psicología con orientación en neurociencia cognitiva

Escuela de Posgrados, Universidad Católica Luis Amigó

Medellín, Colombia

dubis.rinconba@amigo.edu.co

Orcid: https://orcid.org/0000-0002-8322-889X

CvLAC:

https://scienti.minciencias.gov.co/cvlac/visualizador/generarCurriculoCv.do?cod_rh=000099186 4\&lang=null 


\section{Oom Andrés Blanco Belén}

Doctorando en Neurociencia cognitiva aplicada

Escuela de Psicología, Universidad Central del Este

San Pedro de Macoris, República Dominicana

oblanco@uce.edu.do

Orcid: https://orcid.org/0000-0001-7203-729X

\section{Rolan Arcado Correa López}

Doctorando en Psicologia con orientación en neurociencia

Facultad de Psicología y Ciencias Sociales, Universidad Católica Luis Amigó

Bogotá, Colombia

rolan.correalo@amigo.edu.co

Orcid: https://orcid.org/0000-0002-5345-6875

CvLAC:

https://scienti.minciencias.gov.co/cvlac/visualizador/generarCurriculoCv.do?cod_rh=000159536 $\underline{2}$

Recibido: 25 de marzo de 2021

Evaluado: 30 de agosto de 2021

Aprobado: 15 de diciembre de 2021

Tipo de artículo: Revisión

\section{Resumen}

El presente artículo tiene como objetivo presentar los resultados de una revisión narrativa acerca de la relación entre los rasgos de personalidad, la toma de decisiones y la permanencia académica. Para ello se realizó una búsqueda en las bases de datos Science Direct, EBSCO, Proquest, y se utilizaron los descriptores personalidad, rasgos, universitarios, toma de decisiones, permanencia académica. Para el análisis de los textos se tuvieron en cuenta los criterios de revisión de la guía CASPe. Se incluyeron un total de 25 estudios. Se encontró que existe una relación entre la toma de decisiones arriesgada con el factor de afabilidad. Cada nivel de adaptación individual, unido a la motivación de lograr las metas académicas durante el desarrollo de la carrera universitaria, necesitan comprenderse desde una triple perspectiva que no es excluyente. Estas tres variables permiten una visión más específica desde el contexto de toma de decisiones en al ámbito universitario. Finalmente, se plantea la necesidad de profundizar en estudios que involucren las tres variables mencionadas, más allá de rasgos como responsabilidad, neuroticismo y afabilidad, 
y tomar en cuenta los demás factores que se describen en el modelo de los Cinco Factores (Big Five).

Palabras clave: Estudiante universitario; Personalidad; Toma de decisiones.

\begin{abstract}
This article presents a narrative review about the relationship between personality traits, decisionmaking and academic permanence. For this, a search was carried out in the Science Direct, EBSCO, Proquest databases and the descriptors personality, traits, university, decision-making, academic permanence were used. For the analysis of the texts, the review criteria of the CASPe guide were taken into account. A total of 25 studies were included in this review. It was found that there is a relationship between risky decision making and the factor of agreeableness. Each level of individual adaptation, together with the motivation to achieve academic goals during the development of the university career, need to be understood from a triple perspective that is not exclusive. These three variables allow a more specific view from the context of decision-making in the university environment. Finally, the need to delve into studies involving the three variables mentioned, beyond traits such as responsibility, neuroticism and affability, and take into account the other factors that are described in the model of the Five Factors (Big Five).
\end{abstract}

Keywords: University student; Personality; Decision making.

\title{
Introducción
}

La permanencia académica resulta ser un tema de interés, que recientemente ocupa muchas de las acciones emprendidas por instituciones de educación superior, por lo que designan personal y recursos financieros para la implementación de programas con el mismo nombre, muchas veces adscritos a unidades mayores, como las vicerrectorías o unidades encargadas del bienestar universitario (Pineda Báez y Pedraza Ortiz, 2009). Sin embargo, el fenómeno de la deserción, derivado de la no permanencia de los estudiantes en un programa académico en particular, sigue siendo una situación que va en aumento, en la medida que también se asocia con dificultades 
económicas, problemas sociales y otros factores asociados al hecho de permanecer en un programa académico, así como culminar los estudios universitarios, entre ellos los aspectos relacionados con la personalidad y la capacidad para la toma de decisiones.

De esta manera, el presente estudio se centró en la revisión de investigaciones relacionadas con los factores anteriormente mencionados: personalidad y toma de decisiones, los cuales se han definido como determinantes en la permanencia de los estudiantes en un programa académico o una institución, pero sobre los cuales no se han identificado estudios que los relacionen de manera concluyente, generando así un vacío de conocimiento y la necesidad de llevar a cabo investigaciones que permitan identificar la relación o asociación entre la personalidad y la toma de decisiones de tipo académico que faciliten la permanencia del estudiante.

A continuación, y con el ánimo de guiar al lector, se presenta una conceptualización sobre la permanencia académica como constructo, seguido de la definición de rasgos de personalidad, en función de la toma de decisiones, tanto en el aspecto cognitivo como en el comportamental.

\section{Permanencia académica}

La permanencia académica (PA) es descrita como el éxito o nivel de logro que tiene un estudiante para culminar su proceso formativo, y en donde este establece una conducta que permanece en el tiempo (Gutiérrez y Moreno, 2020). El Ministerio de Educación Nacional de Colombia (2009) hace referencia a la permanencia como el sentido de pertenencia, tanto por la elección profesional como por la institución de educación superior en la cual se encuentra vinculado. Por otro lado, Berger, Blanco y Lyon (como se citó en Gutiérrez y Moreno, 2020) mencionan que la permanencia académica es la intención y la acción, por parte de los estudiantes, de mantenerse dentro de la institución y culminar con éxito su proceso académico; lo cual está probablemente determinado por el tipo de personalidad de las y los estudiantes, demostrando así el vínculo psicológico con su entorno y los factores que ayudan, o no, a tomar esta decisión.

Dicho proceso es influenciado por factores externos e internos. En cuanto a los primeros, se encuentran: la relación entre docente y estudiante, el rendimiento académico, los medios educativos y el bienestar universitario, así como las condiciones socioeconómicas, la percepción que se tiene sobre los profesores y la elección del programa -orientación vocacional- (Chalela- 
Naffah et al., 2020; Gómez Cano et al., 2016; Londoño, 2013). Asimismo, según Velázquez Narváez y González Medina (2017) "el entorno familiar y el rendimiento académico previos (...), generan valores y expectativas de éxito que dan como consecuencia la permanencia académica" (p. 120). Lo anterior, implica también un nivel de autopercepción adquirido que se ha formado el individuo a partir del nivel de éxito o fracaso que haya tenido en su recorrido académico, previo al ingreso universitario, lo cual es determinante en los factores internos al momento de decidirse por un programa o carrera académica.

Aunado a lo anterior, Ghignoni (como se citó en Chalela-Naffah et al., 2020) afirma que, si bien el apoyo financiero es un factor importante para continuar con los estudios, este se ve reforzado positivamente si es asociado con la formación de los padres y la motivación que los mismos tienen frente al estudio, lo cual implica una menor probabilidad de que un estudiante decida abandonar o suspender sus estudios.

De esta manera, el factor familiar incide en la permanencia del estudiante, puesto que, si el entorno es negativo, este influirá de manera significativa en el bajo rendimiento académico, el escaso nivel de integración social y el poco compromiso institucional, exponiendo al estudiante a la posibilidad de abandonar los estudios y producir una deserción académica (Spady, como se citó en Rueda et al., 2020).

Respecto a los factores internos, se destacan la motivación personal, la vocación, entendida aquí como la disposición natural hacia el logro de metas y cumplimiento de tareas propias o afines a una determinada elección profesional, ya sea por reconocimiento propio, validación social, o ambas, y el gusto por la carrera (Navarro-Charris et al., 2017; Parada Rico et al., 2017). Además, se ha evidenciado que la satisfacción de los estudiantes, debido a su proceso académico, está relacionada con la PA, puesto que estos logran mantenerse en universidades de alta calidad, en comparación con aquellos estudiantes que observan debilidades en su proceso formativo (ChalelaNaffah et al., 2020; Gómez-Molina et al., 2019; Hernández Enríquez y Vallejo Giraldo, 2017).

\section{Personalidad y toma de decisiones}

Merlyn-Sacoto et al. (2018) afirman que la personalidad es entendida como un grupo de características estables y tendencias que determinan las diferencias psicológicas (pensamientos, 
sentimientos y acciones) de un individuo con otro, que convive en un espacio y tiempo igual, y que no solo responde a las presiones sociales o cambios biológicos. A su vez, Muelas (2014) menciona que la personalidad se produce a partir del intercambio de condiciones como la herencia y el ambiente en el que se desenvuelve el individuo, mediado por el aprendizaje social y las experiencias personales, las cuales cambian a lo largo de la vida.

Estos cambios respectivamente fijos, así como aquellos que no lo son, históricamente han impulsado estudios sobre la personalidad. McDougall (como se citó en Cariaga y Casari, 2014) desarrolló, en 1932, el modelo de los cinco factores (Big five), en el cual, la personalidad se puede entender mediante la expresión de factores, tales como: extraversión, neuroticismo, apertura a la experiencia, agradabilidad y responsabilidad.

Esta teoría tiene un alto grado de consenso para explicar el fenómeno de la personalidad, por lo cual es ampliamente utilizada en investigaciones que den cuenta de la personalidad en diferentes condiciones (Erazo et al., 2019; Gerosa, 2016).

Por su parte, la toma de decisiones (TD) es entendida como una variable asociada al desarrollo cognitivo y social; se define como la capacidad de anticipación, el establecimiento de metas y el diseño de planes, siendo estas funciones ejecutivas de tipo metacognitivo (Acuña et al., 2013; Sarmiento y Ríos, 2017). Según Luna Bernal y Laca Arocena (2014), las funciones metacognitivas incluyen la capacidad de pensamiento abstracto e hipotético, habilidades para asumir un rol y destrezas para planear y prever situaciones futuras.

En esta línea, Buelow \& Cayton (2020) afirman que la personalidad puede influir en la toma de decisiones que los sujetos realizan a lo largo de la vida. Se ha encontrado que existe una relación entre el factor de neuroticismo (alto) y afabilidad (bajo) con la toma de decisiones arriesgadas. Además, se ha evidenciado que el factor neuroticismo, relacionado con la presencia de ansiedad, influye en la toma de decisiones cuando se está bajo presión (Byrne et al., 2015). Al respecto, González et al. (2016) afirman que la habilidad para la toma de decisiones aumenta significativamente, conforme se desarrollan los rasgos que, desde dicho modelo del Big Five, se denominan: cooperación, cordialidad, escrupulosidad, dominancia, dinamismo y perseverancia.

Existen varias hipótesis que determinan las posibles dinámicas que pueden tener influencia dentro del proceso que lleva a la toma de decisiones; por ejemplo, Damasio (1996), con el desarrollo del marcador somático, manifiesta que un cambio a nivel corporal da como respuesta 
un estado emocional, por medio de somatizaciones, y estas pueden afectar directamente a la toma de decisiones (TD). En este sentido, hay presencia de una integración de procesos, tanto cognitivos como emocionales, en aras de definir la relación entre el procesamiento emocional y la capacidad de elección, en función de las posibles consecuencias futuras (Márquez et al., 2013; Reimann \& Bechara, 2010). Estas repuestas somatizadas son dadas por experiencias similares y aprendidas; así pues, su importancia reside en que puede agilizar o facilitar la toma de decisiones.

Por otro lado, estudios realizados han permitido determinar que la intuición es otro de los elementos clave en el proceso de la TD, demostrando que en algunas ocasiones se toma una decisión antes de que esta se haga presente en la consciencia de la persona; es decir, antes de que la persona pueda explicar de manera verbal la razón por la que eligió determinada decisión (Bechara, Damasio, Tranel y Damasio, como se citó en Sarmiento y Ríos, 2017).

\section{Metodología}

Se realizó una investigación de tipo documental sobre los estudios cuyo tema principal fuera la personalidad, la toma de decisiones y la permanencia académica. Para ello, se realizó una revisión narrativa y se buscaron artículos publicados en los últimos 8 años (2012 al 2020) y que estuvieran alojados en las bases de datos EBSCO, Science Direct y Proquest. Se utilizaron los descriptores "personalidad", "big five", "permanencia académica", "toma de decisiones" y "rasgos de personalidad", tomando en cuenta documentos en inglés y español. Asi mismo, se llevó a cabo un proceso de revisión y búsqueda de información secuencial, tomando estudios a partir de sus fuentes primarias, no se exploraron documentos difundidos a través de canales de información comercial.

\section{Criterios para seleccionar los estudios para la revisión}

Para la selección de los estudios, se tuvo en cuenta la clasificación por niveles de evidencia que propone Stetler et al. (1998); así mismo, los artículos de opinión y editoriales, considerados como estudios nivel IV no se tuvieron en cuenta. 


\section{Métodos de revisión}

En una primera fase, para el proceso de sistematización y el análisis de la calidad de los estudios, se revisó la información relacionada al título, los autores y la metodología desarrollada, encontrando 49 artículos con texto completo. Posteriormente, se realizó un análisis crítico de los textos, considerando los criterios de revisión de la guía CASPe; se excluyeron 24 artículos por no presentar dentro de los resultados algún tipo de relación en las variables del estudio (personalidad, toma de decisiones y permanencia académica). Finalmente, en esta revisión se incluyeron un total de 25 estudios. Las fases del proceso de búsqueda, selección, exclusión e inclusión de los estudios se presentan en la Figura 1.

\section{Figura 1}

Método de selección de información

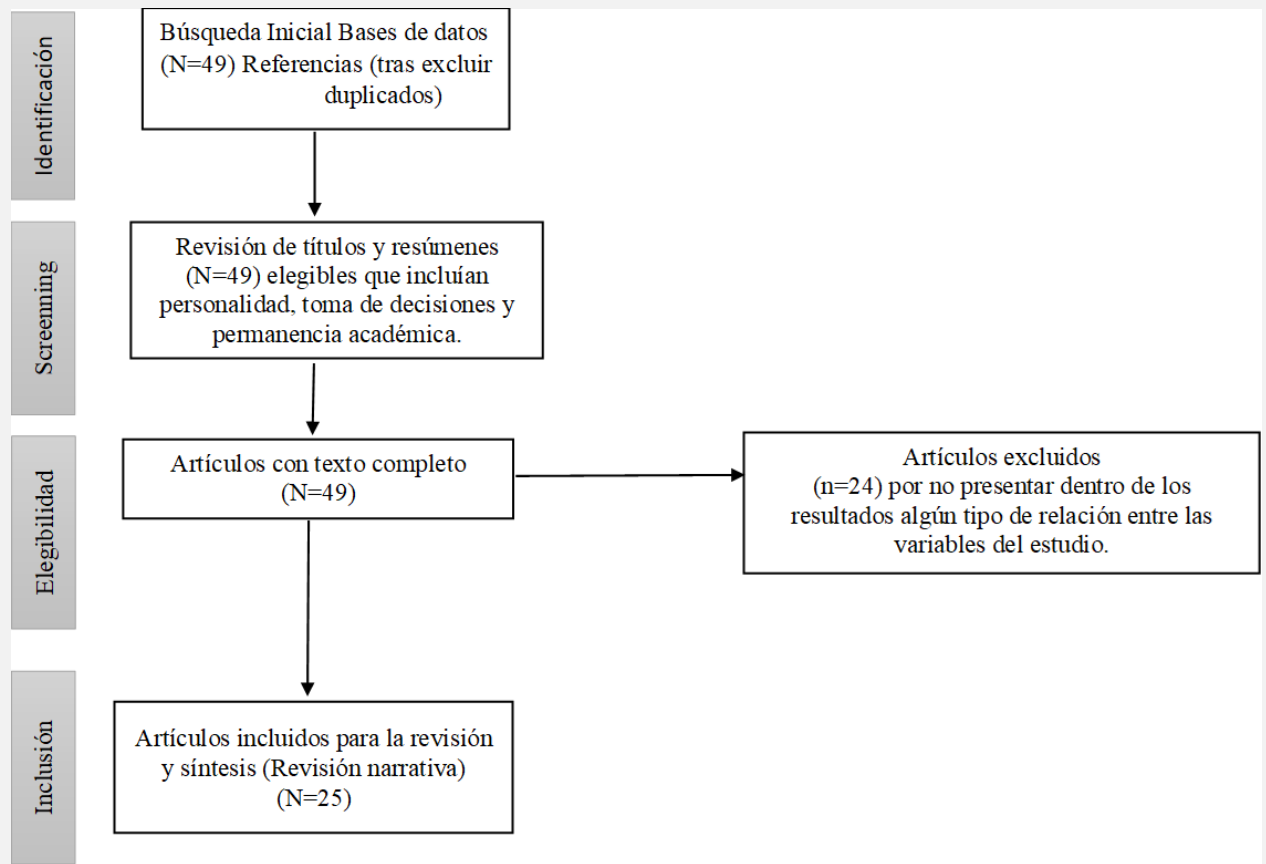

Nota: Adaptada de Rincón et al. (2020). 


\section{Relación entre rasgos | Revista Virtual Universidad Católica del Norte, 65, 263-283 \\ ISSN: 0124-5821 (En línea)}

\section{Resultados}

De un total de 49 artículos se seleccionaron 25 documentos, los cuales incluyeron temas relacionados con personalidad (8), toma de decisiones (6) y permanencia académica (11). Se excluyeron 24 artículos, que no presentaban dentro de los resultados algún tipo de relación entre las variables del estudio, así como aquellos que no presentaban conclusiones respecto a estas, y sus resultados se expresaban como no concluyentes o que requerían una posterior investigación. Por tanto, para la presentación del análisis de los resultados, se tendrán en cuenta dos apartados: 1. Rasgos de personalidad y permanencia académica, y 2 . Toma de decisiones y rendimiento académico.

\section{Rasgos de personalidad y toma de decisiones académicas}

Buelow \& Cayton (2020) encontraron que existe una relación entre la toma de decisiones arriesgada con el factor de afabilidad; esto fue posible por medio de un estudio de tipo correlacional desarrollado en dos fases: la primera, en la que participaron 318 estudiantes de psicología, y a quienes se les aplicó 4 instrumentos de toma de decisiones y el inventario de personalidad Big Five en su versión corta; y la segunda, en la que participaron 518 estudiantes de psicología, a quienes, adicional a los 4 instrumentos que permitieron evaluar toma de decisiones, se les aplicó 3 instrumentos para evaluar los cinco grandes factores de la personalidad (afabilidad, neuroticismo, extraversión, apertura mental, tesón). Sin embargo, no se encontraron diferencias significativas entre los resultados de las pruebas de toma de decisiones y los resultados de las pruebas de personalidad de los cinco grandes factores.

De otro lado, Genise el al. (2018), en un estudio descriptivo-correlacional con una muestra de 1078 estudiantes españoles (585 varones y 493 mujeres), de 3 escuelas públicas y un instituto, evidenciaron, a partir del Big Five, que el factor neuroticismo tiene una correlación negativa con las variables relacionadas con la resiliencia, por lo que se supone que los adolescentes con tendencia neurótica presentan carencias en las habilidades para tratar diversas situaciones de una manera emocionalmente adecuada. 
Por su parte, Delhom et al. (2019), en un estudio correlacional, demostraron que el factor neuroticismo da paso a la inestabilidad emocional; por tanto, el bienestar subjetivo se ve afectado, ya que este depende de una puntuación baja en el factor neuroticismo y alta en el de extraversión (Morán et al., 2017). Por otro lado, Novoa y Barra (2015) plantean que el neuroticismo y la extraversión desempeñan un papel importante en relación con la satisfacción vital de los estudiantes universitarios, permitiendo de esta manera que se pueda predecir su rendimiento y acoplamiento ante la carrera elegida. De este modo, el nivel de adaptación y el deseo de lograr las metas académicas, durante el desarrollo de la carrera universitaria, se vinculan también con rasgos de la personalidad

Según Cuadra-Peralta et al. (2015) y Cupani et al. (2013), la evidencia ha demostrado que existen factores que inciden en la permanencia académica, como lo son el rendimiento académico y el desempeño de los estudiantes. Frente al primero, se encuentra que el factor responsabilidad de la personalidad está directamente relacionado con el rendimiento académico del estudiante, ya que presentó puntuaciones significativas. A su vez, el desempeño del estudiante está relacionado con el factor apertura a la experiencia; por ende, los factores de la personalidad inciden en el comportamiento del estudiante dentro de la vida académica (Gerosa, 2016).

\section{Toma de decisiones y permanencia académica}

Luna Bernal y Laca Arocena (2014), en su estudio descriptivo-correlacional, con una muestra de 992 estudiantes con edades entre los 15 y 19 años, demostraron que los adolescentes, ante la toma de decisiones, frecuentemente responden con estrés de decisión; en este se evidencia un aplazamiento o evitación de dicha decisión (procrastinación), proceso que se podría relacionar con el abandono escolar, es decir, la evitación de la responsabilidad a la cual se enfrentan.

Por otro lado, Luna Bernal et al. (2012), en su estudio correlacional, incluyen también un componente familiar en la toma de decisiones de los adolescentes, demostrando que la implementación de una comunicación, basada en la empatía, la escucha activa, el respeto por la libertad, la comprensión y la transferencia de información entre padres e hijos adolescentes, se relaciona de forma positiva con los adecuados procesos de toma de decisiones; mientras que la 
menor presencia de este factor comunicacional implicaría toma de decisiones menos funcionales, asociándosele también interacciones comunicativas ofensivas y evitativas.

Rueda et al. (2020) afirman que el adecuado funcionamiento de núcleos familiares permite una mayor adaptación por parte del estudiante a la vida académica; además, los primeros semestres son los que presentan mayores dificultades en el ingreso de la vida universitaria. Por tanto, el factor socioeconómico, la dificultad en la elección del programa, padres que no han tenido estudios de educación superior y el interés que poseen por la carrera, son factores que están relacionados con la deserción académica, y que, por tanto, inciden en la toma de decisiones respecto a la no permanencia dentro de la universidad (Londoño, 2013; Regueyra y Rojas, 2013).

Fonseca-Grandón (2018), en un estudio descriptivo, encontró que el currículum (oferta académica) puede estar relacionado con el abandono o la permanencia en la universidad; decisiones que toman los estudiantes a partir de la percepción que tienen del mismo. Dicho estudio fue desarrollado en dos fases: la primera, consistió en indagar por las diferentes variables socioeconómicas y académicas de 1454 estudiantes de primer año de diferentes pregrados; la segunda, profundizó en variables académicas (el currículum universitario, la integración académica y social), preuniversitarias (la predisposición y tendencia) y sociodemográficas. Esta fase estuvo constituida por 171 de estudiantes que cursaron por lo menos un semestre académico y decidieron continuar, y otros que decidieron abandonar la universidad.

En este orden de ideas, se considera que hay una relación existente entre las acciones de permanencia estudiantil y el modelo de abandono estudiantil; es decir, que el factor docente, factor curricular, factor administrativo, factor medios educativos y factor bienestar universitario influyen en la toma de decisiones de un estudiante para permanecer o abandonar el lugar de estudio (Gómez Cano et al., 2016; Gutiérrez y Moreno, 2020). A su vez, Urbina y Ovalles (2016) plantean en su estudio que el papel de los docentes es importante al momento de incentivar al estudiante hacia el aprendizaje; estos se convierten en motivación para que los estudiantes logren el éxito académico y la adaptabilidad en el espacio universitario, desarrollando de esta manera un progreso personal y el alcance de metas.

También, cabe resaltar que si la población estudiantil se encuentra en un proceso de adolescencia pueden verse mayormente expuestos a tomar la decisión de abandonar sus estudios, debido a que esta misma condición les permite desarrollar características de toma de riesgo, 
búsqueda de sensaciones, hipersensibilidad a la recompensa inmediata, bajo control de impulsos y disparidad en la maduración del control cognitivo y afectivo, las cuales dan paso a que las decisiones que tomen bajo esta condición sean impulsivas (Acuña et al. 2013).

Por su parte, Álvarez (2019), en un estudio descriptivo-correlacional, en donde participaron 494 estudiantes, evidenció que en la toma de decisiones influyen las dimensiones cognitiva, social y emocional; no obstante, hay una prevalencia más alta en las dimensiones cognitiva y emocional. A su vez, Sarmiento y Ríos (2017) plantean que, aparte del rol importante que juegan las emociones en los procesos de toma de decisiones, se deben tener en cuenta diferentes variables en este proceso, tales como el procesamiento atencional que se activa por estímulos significativos de la decisión y las experiencias - consecuencias evaluadas y contenidas en la memoria (huellas mnémicas), lo que permite evaluar los escenarios posibles frente a la toma de una decisión.

En consecuencia, el rendimiento académico, la asistencia a clase, el compromiso asumido, la vinculación que el estudiante tenga con la carrera escogida y la motivación y vocación por la misma, determinarán la toma de decisiones para la permanencia académica, donde el estudiante se ve expuesto a elegir la carrera que desarrollará, la motivación y el compromiso que asumirá para llevarla a cabo (Esteban García et al., 2016; Esteban et al., 2017; Mejías Sandia, 2016; Parada Rico et al., 2017). De otro lado, Villasana et al. (2017) plantean que la resiliencia en la adolescencia tiene relación de dependencia con los estilos y estrategias de afrontamiento que se desarrollen.

\section{Discusión}

Este estudio parte con el objetivo de realizar una revisión documental de investigaciones relacionadas con la permanencia académica (PA), la personalidad y la toma de decisiones (TD), para determinar una posible relación entre estas variables que permita la comprensión integral de los aspectos que no son excluyentes entre sí. De esta manera, según lo encontrado, es pertinente realizar las siguientes apreciaciones.

En primer lugar, entendiendo la personalidad como un grupo de características estables y tendencias que determinan las diferencias psicológicas (pensamientos, sentimientos y acciones) de un individuo a otro (Merlyn-Sacoto et al., 2018), y partiendo de que la toma de decisiones es la capacidad de anticipación, el establecimiento de metas y el diseño de planes (Acuña et al., 2013; 
Arévalo-Avecillas et al., 2019; Sarmiento y Ríos, 2017) se encontró que algunos rasgos de la personalidad, tales como afabilidad y neuroticismo, están asociados con la toma de decisiones, pero que es necesario comprenderla desde todos los rasgos posibles para lograr una aprehensión y, posteriormente, una comprensión en valores significativos acerca de la dinámica de personalidad implicada en la permanencia académica.

Buelow \& Cayton (2020) hallaron que la afabilidad se relaciona con la toma de decisiones arriesgada; esta se puede entender como aquella característica de personalidad marcada por amabilidad, simpatía, altruismo, sensibilidad y confianza ante los demás, y los aspectos que componen su realidad, por lo que una persona con estas características probablemente tomará una decisión arriesgada, sin realizar un proceso de valoración de las consecuencias o efectos que esta pueda generar; además, permite establecer elementos externos pertenecientes al contexto universitario que pueden generar, indistintamente del programa académico, un clima que ocasione deserción académica.

Por su parte, Genise et al. (2018) evidenciaron que el factor neuroticismo, al estar determinado por la inestabilidad emocional, se relaciona de manera negativa con el proceso de TD, esto quiere decir que a mayor inestabilidad emocional existe una menor capacidad para la TD; por ejemplo, una persona que experimenta situaciones de inseguridad presentará mayores dificultades en el momento en que requiera tomar una decisión.

En segundo lugar, la relación entre PA y TD está mediada por los factores externos e internos que influyen sobre esta última, siendo claves en la decisión de permanecer o no en la universidad. En cuanto a los factores externos, se encontró que el rendimiento académico (previo y actual), la relación que se establezca con los órganos administrativos y el personal docente, así como el entorno familiar y las condiciones socioeconómicas con las cuales cuenta la persona están estrechamente relacionadas con la PA (Gómez Cano et al., 2016; Londoño, 2013). La universidad debe considerar entonces un ambiente relacional que permita en aspectos generales, la expresión sana e integral de cada tipo de personalidad y su vinculación con los estudiantes, potenciando aquellos casos que permitan una toma de decisiones asertiva y minimice el absentismo y deserción en las aulas. Respecto a los factores internos, se evidenció que la vocación, compromiso y motivación frente a la carrera escogida también son determinantes para permanecer en un programa académico (Parada Rico et al., 2017). 
En relación con lo mencionado anteriormente, cabe resaltar también que el factor responsabilidad de la personalidad se relaciona con la permanencia y el rendimiento académico (Cuadra-Peralta et al., 2015), razón por la cual se incluye en los aspectos internos que permean el proceso de TD; por tanto, se podría inferir que este factor, al hacer parte de la estructura del sujeto, tiene mayor peso y le posibilitará tomar la decisión de permanecer, por encima de aquellos factores externos.

De otro lado, es importante tener en cuenta que muchos estudiantes inician la vida universitaria entre los 15 y 19 años, y por ello, estos pueden responder con estrés ante la toma de decisiones, por lo que algunos pueden evitarla o aplazarla (Luna Bernal y Laca Arocena, 2014). En palabras de Damasio (1996), un cambio a nivel corporal da como respuesta un estado emocional que se somatiza y, por tanto, puede afectar la toma de decisiones. Es decir, el estrés incide en la forma en que unas personas u otras toman una decisión.

Tomando en cuenta lo anterior, es importante resaltar lo hallado por Luna Bernal et al. (2012), en donde se expone la importancia de aspectos al interior de la familia y que se asocian con los estilos de comunicación, empatía, escucha activa, comprensión y libertad entre padres e hijos, y que se relacionan con el proceso de TD y elección de una carrera, lo que va a verse reflejado después en la PA. Por otro lado, Rueda et. al. (2020) destacan la influencia que variables de tipo familiar, en aspectos como el nivel educativo de los padres y nivel socio-económico del núcleo familiar, también influyen dentro de la TD frente a la carrera que se elige, adaptación y PA de los estudiantes durante los primeros semestres, de tal forma que al reconocer estos factores e incluirlos en futuras investigaciones se ampliaría el espectro en el que se podrían analizar los datos de una muestra, esperando encontrar otro tipo de relaciones adicionales en el proceso de TD y PA.

Así mismo, se debe tomar en cuenta el papel que cumplen las emociones dentro del proceso de TD y PA en estudiantes, ya que como lo resalta Sarmiento y Ríos (2017), y lo confirma Álvarez (2019), estas además ejercen una influencia en aspectos de tipo cognitivo y emocional, al tiempo que se encuentran relacionadas con procesos como la atención, que luego generará una huella de memoria. Así las cosas, sería necesario considerar la necesidad de evaluar, además de aspectos relacionados con la personalidad y la TD, elementos de tipo cognitivo que permitieran describir el desempeño de cada participante, buscando una relación con la TD y factores a nivel de personalidad. 
En este mismo sentido, profundizar en la influencia de las emociones y de estilo atribucional, que contribuyen a generar expectativas y conclusiones de su rendimiento académico, para decidir si permanecen o no en la universidad.

Sin embargo, los estudios encontrados a partir de la revisión documental no muestran resultados concluyentes sobre la relación entre las tres variables mencionadas al inicio de este apartado (personalidad, toma de decisiones y permanencia académica); solo se evidenciaron relaciones entre algunas características de dichas variables; por ejemplo, en cuanto a los rasgos de personalidad, las investigaciones incorporaron rasgos como responsabilidad, neuroticismo y afabilidad, pero no tuvieron en cuenta los demás factores que se describen en el modelo del Big Five.

Finalmente, y no menos importante, saber administrar evaluaciones que vinculen el rendimiento académico con las funciones ejecutivas para obtener un mejor pronóstico de permanencia universitaria y complemente la toma de decisiones; es un gran campo de investigación que debe ser tomado en cuenta, más allá de las pruebas de CI. Las competencias cognitivas, técnicas, emocionales y relacionales son la expresión del procesamiento integral, como producto de la actividad mental sobre la base de la personalidad que vincula y la expresa de forma peculiar.

Lo anterior, sugiere la necesidad de realizar investigaciones que tengan en cuenta todos los niveles de las tres variables; así mismo, es importante revisar la pertinencia del constructo personalidad desde el modelo del Big Five, específicamente cuando se aplica en procesos de investigación. De igual forma, es necesario precisar el concepto de PA, debido a que suele ser llamado de manera indistinta y confundirse con aspectos como desempeño académico, rendimiento académico, éxito académico, entre otros, desconociendo que la permanecía académica hace referencia al hecho de permanecer o quedarse dentro de un programa académico, independiente del desempeño o rendimiento que se tenga.

\section{Conclusiones}

A partir de los resultados de esta revisión, es posible concluir que los rasgos de personalidad, como la afabilidad y el neuroticismo, pueden influir en la PA de los estudiantes, en 
tanto las investigaciones señalan una relación positiva entre la afabilidad y la TD, contrario a lo que ocurre con el neuroticismo, en tanto, se inclina más hacia la inestabilidad emocional.

Así mismo, se resaltan aspectos adicionales que influyen en la interacción de las variables revisadas (PA, TD y personalidad), como por ejemplo los factores externos dentro de los cuales es posible identificar la relación que los estudiantes establecen con las áreas administrativas de las universidades, al tiempo que factores internos como la vocación, motivación y compromiso del estudiante, reconociendo su interacción directa como elementos que influyen en la PA de los jóvenes estudiantes, a lo cual, a su vez, se le suman las edades en las cuales ellos inician su vida universitaria y las decisiones que ellos toman, mediadas por las emociones y el aporte de la familia dentro de las mismas.

En suma, esta investigación abre la posibilidad para estudios posteriores de carácter empírico y con diseño experimental, con el ánimo de conocer no solo la relación de las variables como se hizo en este estudio, sino la posible influencia que tienen los rasgos de personalidad en la toma de decisiones y luego en la toma de decisiones académicas. Lo anterior, redundando en la posibilidad de generar programas de permanencia académica, basados en resultados de investigación, los cuales puedan ser realmente efectivos y pertinentes a las dinámicas de las instituciones educativas en países latinoamericanos.

\section{Referencias}

Acuña, I., Castillo, D., Bechara, A., y Godoy, J. C. (2013). Toma de decisiones en adolescentes: rendimiento bajo diferentes condiciones de información e intoxicación alcohólica. International Journal of Psychology and Psychological Therapy, 13(2), 195-214. https://www.redalyc.org/pdf/560/56027416004.pdf

Álvarez, J. (2019). Las dimensiones cognitiva, emocional y social en la toma de decisiones en el alumnado de educación secundario. REOP- Revista Española de Orientación y Psicopedagogía, 30(3), 140-153. https://doi.org/10.5944/reop.vol.30.num.3.2019.26277

Arévalo-Avecillas, D., Padilla-Lozano, C., Pino, R., y Cevallos, H. (2019). Los Dominios de la Personalidad y su Relación con el Estilo de Liderazgo Transformacional. Información Tecnológica, 30(3), 237-248. https://dx.doi.org/10.4067/S0718-07642019000300237 
Buelow, M. T., \& Cayton, C. (2020). Relationships between the big five personality characteristics and performance on behavioral decision making tasks [Relaciones entre las cinco grandes características de la personalidad y el desempeño en las tareas de toma de decisiones conductuales]. Personality and Individual Differences, 160, Artículo 109931. https://doi.org/10.1016/j.paid.2020.109931

Byrne, K., Silasi-Mansat, C., \& Worthy, D. (2015). Who chokes under pressure? The Big Five personality traits and decision-making under pressure [¿Quién se ahoga bajo presión? Los cinco grandes rasgos de personalidad y toma de decisiones bajo presión]. Personality and individual differences, 74, 22-28. https://doi.org/10.1016/j.paid.2014.10.009

Cariaga, M. L., y Casari, L. (2014). La incidencia de rasgos de personalidad en estudiantes avanzados de Psicología hacia la elección de una línea teórica. Psicogente, 17(32), 379396. http://www.scielo.org.co/pdf/psico/v17n32/v17n32a10.pdf

Chalela-Naffah, S., Valencia-Arias, A., Ruiz-Rojas, G., y Cadavid-Orrego, M. (2020). Factores psicosociales y familiares que influyen en la deserción en estudiantes universitarios en el contexto de los países en desarrollo. Revista Lasallista de Investigación, 17(1), 103-115. https://doi.org/10.22507/rli.v17n1a9

Cuadra-Peralta, A., Veloso Besio, C., Marambio-Guzmán, K., y Tapia Henríquez, C. (2015). Relación entre rasgos de personalidad y rendimiento académico en estudiantes universitarios. Interciencia, 40(10), 690-695. https://www.interciencia.net/wpcontent/uploads/2017/10/690-C-CUADRA-6.pdf

Cupani, M., Garrido, S. J., y Tavella, J. (2013). El modelo de los cinco factores de personalidad: contribución predictiva al rendimiento académico. Revista de Psicología, 9(17). https://repositorio.uca.edu.ar/handle/123456789/6232

Damasio, A. R. (1996). The somatic marker hypothesis and the possible functions of the prefrontal cortex [La hipótesis del marcador somático y las posibles funciones de la corteza prefrontal]. Philosophical Transactions of the Royal Society of London. Series B: Biological Sciences, 351(1346), 1413-1420. https://doi.org/10.1098/rstb.1996.0125

Delhom, I., Satorres, E., y Meléndez, J. C. (2019). ¿Están los rasgos de personalidad asociados al bienestar psicológicos? Escritos de Psicología, 12(1), 1-8. https://dx.doi.org/10.5231/psy.writ.2019.0107 
Erazo, G., Santos, D., Pilco, A., y Llanga, J. (2019). Análisis de validez y confiabilidad del Testst de personalidad Big Five en estudiantes universitarios de Ecuador. Revista Puce, (109), 25-44. https://doi.org/10.26807/revpuce.v0i109.244

Esteban García, M., Bernardo Gutiérrez, A. B., y Rodríguez-Muñiz, L. J. (2016). Permanencia en la universidad: la importancia de un buen comienzo. Aula abierta, 44(1), 1-6. https://reunido.uniovi.es/index.php/AA/article/view/11659

Esteban, M., Bernardo, A., Tuero, E., Cervero, A., y Casanova, J. (2017). Variables influyentes en progreso académico y permanencia en la universidad. European Journal of Education and Psychology, 10(2), 75-81. https://www.redalyc.org/pdf/1293/1293533356005.pdf

Fonseca-Grandón, G. R. (2018). Trayectorias de permanencia y abandono de estudios universitarios: una aproximación desde el currículum y otras variables predictoras. $\begin{array}{llll}\text { Educación } & y & \text { Educadores, } & \text { 21(2), }\end{array}$ https://educacionyeducadores.unisabana.edu.co/index.php/eye/article/view/8523/4868

Genise, G., Genise, N., Gómez Darriba, M., Humeniuk, A., y Jordán Muiños, F. (2018). Relación entre resiliencia psicológica y factores de personalidad en población adolescente. Revista Latinoamericana de Ciencia Psicológica, 10(3), 1-16. https://dialnet.unirioja.es/servlet/articulo?codigo $=7324845$

Gerosa, F. (2016). Personalidad, Autoestima y Desempeño Académico. Una aproximación complementaria a partir de la Teoría de los Cinco Factores de la personalidad. $\{P S O C I A L\}$, 2(2), 46-53.

https://publicaciones.sociales.uba.ar/index.php/psicologiasocial/article/view/2146

Gómez Cano, C. A., Sánchez Castillo, V., y Jiménez Zapata, E. M. (2016). Factores endógenos relacionados con la permanencia irregular en las IES, una aproximación conceptual. Revista de Investigación en Administración, Contabilidad, Economía y Sociedad, 4(5), 285-306. https://ojs.tdea.edu.co/index.php/encontexto/article/view/379/397

Gómez-Molina, S., Palacios-Moya, L., Berrio-Calle, J. E., Gaviria-Zapata, S., Quiceno-Merino, L. M., \& Figueroa-Álvarez, P. (julio-diciembre, 2019). Modelo de satisfacción de egresados universitarios: un estudio de caso. Revista CEA, 5(10), 49-68. https://doi.org/10.22430/24223182.1443 
González, J., Gárces, E., López, C., y Zapata, J. (2016). Personalidad y estilos de toma de decisiones en la práctica deportiva. Revista Iberoamericana de Psicología del Ejercicio y el deporte, 11(1), 107-112. https://www.redalyc.org/pdf/3111/311143051012.pdf

Gutiérrez, A. D., y Moreno, L. M. (enero-abril, 2020). Correlación entre acciones de permanencia estudiantil y la resonancia de marca en una institución de educación superior. Revista Logos Ciencia \& Tecnología, 12(1), 85-94. https://doi.org/10.22335/rlct.v12i1.1098

Hernández Enríquez, C., y Vallejo Giraldo, F. (2017). Acompañamiento tutorial en la Universidad de Antioquia. Revista Virtual Universidad Católica Del Norte, (52), 3-22. https://revistavirtual.ucn.edu.co/index.php/RevistaUCN/article/view/941/1388

Londoño, F. L. (2013). Factores de riesgo presentes en la deserción estudiantil en la Corporación Universitaria Lasallista. Revista Virtual Universidad Católica del Norte, (38), 183-194. https://revistavirtual.ucn.edu.co/index.php/RevistaUCN/article/view/413

Luna Bernal, A. C., Laca Arocena, F. A., y Cedillo Navarro, L. I. (2012). Toma de decisiones, estilos de comunicación en el conflicto y comunicación familiar en adolescentes bachilleres. Enseñanza e Investigación en Psicología, 17(2), 295-311. https://www.redalyc.org/pdf/292/29224159014.pdf

Luna Bernal, A. C., y Laca Arocena, F. A. V. (2014). Patrones de toma de decisiones y autoconfianza en adolescentes bachilleres. Revista de Psicología, 32(1), 39-65. https://www.redalyc.org/pdf/3378/337831261002.pdf

Márquez, M., Salguero, P., Paíno, S., y Alameda, J. (2013). La hipótesis del Marcador Somático y su nivel de incidencia en el proceso de toma de decisiones. Revista Electrónica de $\begin{array}{llll}\text { Metodología Aplicada, } & \text { 18(1), }\end{array}$ https://reunido.uniovi.es/index.php/Rema/article/view/9827

Mejías Sandia, C. (julio-septiembre, 2016). Formación identitaria como eje articulador de permanencia y éxito académico de estudiantes de educación superior. Interações (Campo Grande), $17(3)$, $475-486$. https://www.scielo.br/j/inter/a/RqkmRq4bHCHTbYBJvzbXbQg/?format=pdf\&lang=es

Merlyn-Sacoto, M. F., Acurio-Velasco, C. V., Cabezas-Guerra, C. B., Orbe-Nájera, C. E., y RieraVásquez, W. L. (2018). Rasgos de personalidad que afectan el desempeño de los profesores 
de la PUCE matriz en las funciones de docencia e investigación. Estudios pedagógicos (Valdivia), 44(1), 331-349. http://dx.doi.org/10.4067/S0718-07052018000100331

Ministerio de Educación Nacional. (2009). Deserción estudiantil en la educación superior colombiana. Metodología de seguimiento, diagnóstico y elementos para su prevención. Imprenta Nacional de Colombia.

Morán, M. C., Fínez, M. J., y Fernández-Abascal, E. G. (2017). Sobre la felicidad y su relación con tipos y rasgos de personalidad. Clínica y Salud, 28(2), 59-63. https://doi.org/10.1016/j.clysa.2016.11.003

Muelas, Á. (2014). Influencia de la variable de personalidad en el rendimiento académico de los estudiantes cuando finalizan la Educación Secundaria Obligatoria (ESO) y comienzan Bachillerato. Historia y Comunicación Social, 18, 115-126. https://doi.org/10.5209/rev_HICS.2013.v18.44230

Navarro-Charris, N., Redondo-Bilbao, O., Contreras-Salinas, J., Romero-Díaz, C., y AndreisZapata, A. (2017). Permanencia y deserción versus autoeficacia de estudiantes universitarios: un desafío de la calidad educativa. Revista Lasallista de Investigación, 14(1), 198-206. https://doi.org/10.22507/rli.v14n1a17

Novoa, C., y Barra, E. (2015). Influencia del apoyo social percibido y los factores de personalidad en la satisfacción vital de estudiantes universitarios. Terapia Psicológica, 33(3), 239-245. https://scielo.conicyt.cl/pdf/terpsicol/v33n3/art07.pdf

Parada Rico, D. A., Correa Suárez, L. Y., y Cárdenas González, Y. F. (2017). Factores relacionados con la permanencia estudiantil en programas de pregrado de una universidad pública. Investigación en Enfermería: Imagen y Desarrollo, 19(1), 155-170. https://doi.org/10.11144/Javeriana.ie19-1.frpe

Pineda Báez, C., y Pedraza Ortiz, A. (septiembre-diciembre, 2009). Programas exitosos de retención estudiantil universitaria: las vivencias de los estudiantes. Revista Virtual $\begin{array}{llll}\text { Universidad Católica } \quad \text { Del } & \text { Norte, } & \text { (28), } & \text { 1-30. }\end{array}$ https://revistavirtual.ucn.edu.co/index.php/RevistaUCN/article/view/88

Regueyra, G., y Rojas, C. (2013). Una mirada a la permanencia de la población estudiantil de trabajo social. Actualidades Investigativas en Educación, 13(3), 83-108. https://www.redalyc.org/pdf/447/44729878004.pdf 
Reimann, M., \& Bechara, A. (2010). The somatic marker framework as a neurological theory of decision-making: Review, conceptual comparisons, and future neuroeconomics research [El marco de los marcadores somáticos como teoría neurológica de la toma de decisiones: revisión, comparaciones conceptuales e investigación futura en neuroeconomía]. Journal of Economic Psychology, 31(5), 767-776. https://doi.org/10.1016/j.joep.2010.03.002

Rincón, B. D., Restrepo, E. S., Correa, P. L., Ospina, D. J., y Montoya, A. D. (2020). Trastorno dismórfico corporal: una revisión de las dimensiones clínicas, neurobiología y neuropsicología. Cuadernos Hispanoamericanos de Psicología, 19(1), 1-21. https://doi.org/10.18270/chps..v19i1.3133

Rueda, S. M., Urrego, D., Páez, E., Velásquez, C., y Hernández, E. M. (2020). Perfiles de riesgo de deserción en estudiantes de las sedes de una universidad colombiana. Revista De Psicología, 38(1), 275-297. https://doi.org/10.18800/psico.202001.011

Sarmiento, L. F., y Ríos, J., A. (2017). Bases neurales de la toma de decisiones e implicación de las emociones en el proceso. Revista Chilena de Neuropsicología, 12(2), 32-37. https://www.redalyc.org/pdf/1793/179354005006.pdf

Stetler, C. B., Morsi, D., Rucki, S., Broughton, S., Corrigan, B., Fitzgerald, J., Giuliano, K., Havener, P., \& Sheridan, E. A. (1998). Utilization-focused integrative reviews in a nursing service [Revisiones integradoras centradas en la utilización en un servicio de enfermería]. Applied Nursing research, 11(4), 195-206. https://doi.org/10.1016/S0897-1897(98)80329$\underline{7}$

Urbina, J. E., y Ovalles, G. A. (2016). Abandono y permanencia en la educación superior: Una aplicación de la Teoría Fundamentada. Sophia, 12(1), 27-37. https://doi.org/10.18634/sophiaj.12v.1i.290

Velázquez Narváez, Y., y Gonzáles Medina, M. (2017). Factores asociados a la permanencia de estudiantes universitarios: caso UAMM-UAT. Revista de Educación Superior, 46(184), 117-138. https://doi.org/10.1016/j.resu.2017.11.003

Villasana, M., Alonso, J., y Ruiz, M. (2017). Procesos de afrontamiento y factores de personalidad como predictores de la resiliencia en adolescentes: validación de un modelo estructural. Revista de psicodidáctica, 22(2), 93-101. https://doi.org/10.1016/j.psicod.2017.05.004 